\title{
A structured needs assessment does not improve clinical outcomes for clients under the care programme approach
}

Marshall M, Lockwood A, Green $G$, et al. Systematic assessments of need and care planning in severe mental illness: cluster randomised controlled trial. Br J Psychiatry 2004;185:163-8.

\section{Do clients benefit if formulation of their care plan is based on a formal, standardised assessment of need?}

\section{METHODS}

口

Design: Randomised controlled trial.

X?

Allocation: Not stated

Blinding: Single blinded (assessor at 12 months was blind to group allocation).

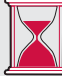

Follow up period: 12 months.

Setting: National Health Service Trusts in urban areas of northwest England; October 1998 to October 2000

Patients: 304 participants (in 72 clusters of up to six people under a given care coordinator); being cared for in the community under care programme approach (CPA); meeting Goldman's criteria for severe mental disorder.

R

Intervention: The trial comprised two parallel experiments. In the first, clusters were randomised to structured assessment or control (experiment 1). In the second, individuals under a single care coordinator were randomised to structured assessment or control (experiment 2). Both groups received a baseline needs assessment. In intervention groups, the results were fed back to the care coordinator, detailing the interventions that would be appropriate for the client and how to obtain them in the locale. In the control group, the results of needs assessment were not made available to the care coordinator to guide management.

Outcomes: Mental state (Brief Psychiatric Rating Scale, BPRS); social functioning (World Health Organisation Psychiatric Disability Assessment Schedule, WHODAS); quality of life (Manchester Short Assessment of Quality of Life, MANSA); patient satisfaction (Client Satisfaction Index); met need (baseline needs that were no longer present at follow up).

$\vec{\square}$

\section{MAIN RESULTS}

Results were combined from both experiments. At 12 months there was no significant difference between intervention and control groups in mental state, social functioning, quality of life, or proportion of needs met (mean BPRS: 5.56 with intervention $v 5.19$ with control; difference $0.34,95 \% \mathrm{CI}-0.67$ to 1.35 ; $\mathrm{p}=0.5 \mathrm{l}$; mean WHODAS: 0.68 with intervention $v 0.69$ with control; difference $0.016,95 \% \mathrm{CI}-0.13$ to 0.16 ; $\mathrm{p}=0.83$; mean MANSA: 4.66 with intervention $v 4.58$ with control; difference 0.032 , 95\% CI -0.147 to $0.210 ; \mathrm{p}=0.73$; met needs $89 \%$ with intervention $v 87 \%$ with control; OR $1.28,95 \%$ CI 0.57 to $2.88 ; \mathrm{p}=0.52$ ).

\section{CONCLUSIONS}

Standardised needs assessment does not improve clinical outcomes for clients under the care programme approach.

For correspondence: Professor M Marshall, Academic Unit, Lancashire Care Trust, Royal Preston Hospital, Fulwood, Preston PR2 9HT, UK

Sources of funding: Wellcome Trust grant.

NOTES

In the individually randomised experiment (experiment 2 ), the care of participants may have been influenced by the coordinator's knowledge of the structured assessment techniques. However, this hypothesis was tested statistically and the authors felt it was legitimate to combine results from the two experiments.

\section{Commentary}

$T$ he aim of this paper was to assess effectiveness of a standardised needs assessment in reducing the rate of unmet need for service among individuals with severe mental illness in community care. The study found no intervention effect after 12 months - the rate of met need was similar across the study groups, as were rates of improvement in assessed outcomes.

These results are provocative in two areas. First, the performance of the care coordinators does not improve when a more systematic needs assessment strategy is used. Given that $30 \%$ of care coordinators did not participate, it is possible that the study attracted providers who are more committed and competent, hence less likely to benefit from the intervention. However, approaches for assessing met need other than receipt of the service may have been more sensitive to provider variation-for example, amount or appropriateness of care. ${ }^{1}$

The second finding, that client outcomes were unaffected by the intervention, follows given that the rate of unmet need was similar between the two groups. However, it does raise the question of whether to expect a link between a process intervention and outcome. Previous studies have found that improved service delivery (including reducing the rate of unmet need) does not lead to better clinical outcomes. ${ }^{2}{ }^{3}$ One explanation is that outcome is affected by the quality, not just the type, of care provided, an explanation also considered by the authors of this study who recommended a narrower focus on unmet need for evidencebased interventions. It is a consistent challenge in the field to identify the critical ingredients of the care process. Studies of effective treatment models, such as assertive community treatment, are now trying to identify the key ingredients. ${ }^{4}$ When asked, consumers tend to value relational over substantive aspects of the care experience, ${ }^{56}$ and several studies have linked therapeutic alliance with outcome.

This study has produced some unexpected findings and raised important questions about what elements of the care experience are most important to standardise and monitor in the aim of improving client outcome.

Janet Durbin, PhD

Research Scientist, Health Systems Research and Consulting Unit, Centre for Addiction and Mental Health and University of Toronto, Toronto,

Canada

1 Crane-Ross D, Roth D, Lauber B. Consumers and case managers' perceptions of mental health and community support needs. Community Ment Health J 2000;36:161-78.

2 Bickman L. A continuum of care. More is not always better. Am Psychol 1996:51:689-701.

3 Lehman AF, Postrado LT, Roth D, et al. Continuity of care and client outcomes in the Robert Wood Johnson Foundation program on chronic mental illness. Milbank Q 1994;72:105-22.

4 Rapp CA. The active ingredients of effective case management: a research synthesis. Community Ment Health J 1998;34:363-80.

5 McGrew J, Wilson R, Bond G. Client perspectives on helpful ingredients of assertive community treatment. Psychiatr Rehabil J 1996;19:14-21.

6 Ware NC, Tugenberg T, Dickey B. Practitioner relationships and quality of care for low-income persons with serious mental illness. Psychiatr Serv 2004;55:555-9.

7 Neale MS, Rosenheck RA. Therapeutic alliance and outcome in a VA intensive case management program. Psychiatr Serv 1995;46:719-21. 